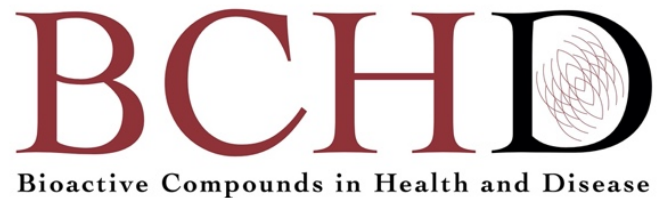

\title{
Dietary deficiencies exacerbate disparity in COVID-19 and nutrition recommendations for vulnerable populations
}

\section{Pooja Polamarasetti ${ }^{1 *}$, Danik Martirosyan²}

Wayne State University School of Medicine, Detroit, MI 48202, USA; ${ }^{2}$ Functional Food Center/Functional Food Institute, Dallas, TX 75252, USA

*Corresponding Author: Pooja Poamarasetti, Wayne State University School of Medicine, Detroit, MI 48202, USA

Submission Date: September $10^{\text {th }}, 2020$; Acceptance Date: November $9^{\text {th }}, 2020$; Publication Date: November $16^{\text {th }}, 2020$

Please cite this article as: Polamarasetti P. Dietary deficiencies exacerbate disparity in COVID-19 and nutrition recommendations for vulnerable populations. Bioactive Compounds in Health and Disease 2020. 3(11): 204-213. DOI: https://www.doi.org/10.31989/bchd.v3i11.759

\section{ABSTRACT}

Marginalized populations face health disparity due to multiple different avenues during the 2020 COVID-19 pandemic:

1. Direct COVID-19 infection and hospitalization,

2. Poor overall health and nutrition leading to comorbidity and worsened outcomes, and

3. Difficult social conditions such as workplace hygiene, living conditions, and transportation.

These three identified issues are cyclical in nature and both cause and affect the others. This article studies each of these factors using a literature review, especially in the experience of certain demographics, including elderly, low-income, racial minorities, urban, and institutionalized persons. We then delve into recommendations that can be considered for each of these issues and specific populations. Recommendations can be given to policymakers, public health officials, and epidemiologists as well as basic science researchers and food producers to attack the disparity from both sides. These findings and ideas may be used to improve both nutrition accessibility as well as COVID-19 disparity for these most vulnerable populations. 
Keywords: COVID-19, SARS-CoV-2, coronavirus, deficiency, elderly, urban, race, socioeconomic status, disparity, nutrition, diet

CFFC 2020. This is an Open Access article distributed under the terms of the Creative Commons Attribution 4.0 License (http://creativecommons.org/licenses/by/4.0)

\section{INTRODUCTION}

COVID-19 disease caused by the novel coronavirus SARS-CoV-2 emerged in December 2019 and quickly began to spread internationally, becoming classified as a pandemic in March 2020 [1]. By September 10 28,316,377 cases and 913,257 deaths have been reported worldwide [2]. Almost every person in the world has either been infected or otherwise affected (through infection of family or friends, school and work closures, stay-at-home orders, etc.).

Interestingly, there is growing evidence that theSARSCoV-2 virus disproportionately targets certain endangered populations, such as low-income, racial minorities, and older age groups. Apart from viral disease, these marginalized groups are also most likely to face a host of other issues regarding food shortages and accessibility. This article will examine each of these issues and study the causes of the increased infection rate in vulnerable groups, especially as influenced by nutritional deficiencies in these populations.

Malnutrition and poor diet can increase susceptibility to the SARS-CoV-2 virus in two ways: by leading to chronic diseases or comorbidities that weaken the body, or by directly affecting the body's immunity and ability to fight off viruses. Following an examination of these disparities, we will expand to suggestions for the vulnerable to obtain important nutritional compounds and improve health.

Risk and Intersectionalities: Although COVID-19 was initially known as "the great equalizer" because it infected populations worldwide, epidemiology trends illustrate that the disease is actually anything but.

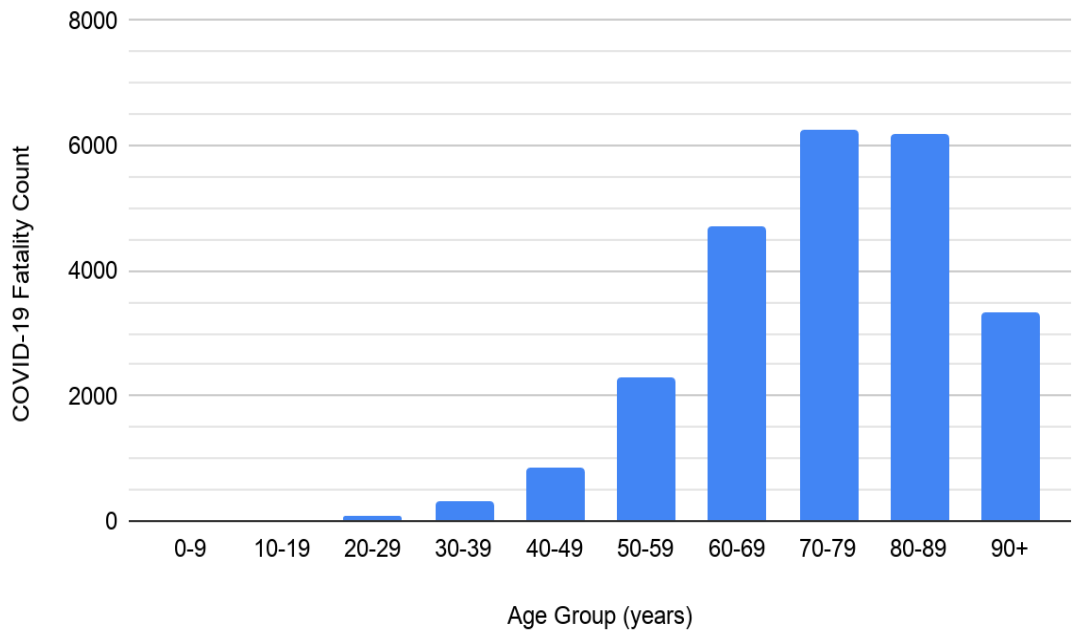

Figure 1. New York State COVID-19 Fatalities by Age Group. Adapted with permission from Functional Food Center, 2020, pp. $231[5]$. 
Different intersectionalities and identities can amplify health disparities in COVID-19 disease rates between different populations. Widely regarded as the highest indication of susceptibility to infection is age. Elderly people are at huge risk of contracting SARS-CoV-2, and suffering poor health outcomes once infected. A recent clinical study of 1,591 COVID19 patients in Italy found that the mean age of patients was 63 years, almost two decades higher than the average population age [3]. In fact, immunity has been proven to decline with age due to high levels of chronic inflammation, dysregulation of innate immunity, oxidative stress, and altered T-cell and Bcell development [4]. This phenomenon places people at or above age 65 at a huge disadvantage when fighting viruses like SARS-CoV-2. The figure below shows the increase in deaths attributed to COVID-19 based on patient age in New York State [5]. These deaths show an overall direct relationship, although they begin to dwindle at high ages due to lower numbers of the population that survives past age 80 [6].

Another huge risk-factor to poor health outcomes involved with COVID-19 is socioeconomic status. Lower income families may have irregular access to medical care and may be less willing to seek care at first suspicion of manifestation, ignoring symptoms and attempting to recover from home or continue working. The stigma associated with testing positive is also a deterrent to openly confirming COVID-19 status to social and workplace circles. Institutionalized persons are also highly vulnerable, such as those in refugee or detainment camps, correctional facilities, rehabilitation centers, or mental health institutions. In recent months, college institutions and dorm rooms have also served as community epicenters of the disease [7]. Poor or crowded living conditions may create both exacerbation of symptoms in the individual patient and spread the virus further through these populations.

Due to high population density and poverty levels, susceptibility to the SARS-CoV-2 virus is highest in busy urban centers where people might still work during a mass quarantine [8]. During the COVID19 pandemic in the United States, any non-essential employee that was able to be told to work remotely. However, workplace data in the figure below shows that as income goes down, so does the likelihood that the job can be done remotely [9]. Although the data in this figure is from past (non-pandemic) years, the relationship between income level and working from home still stands. Many of society's poorest are being sent in to work on frontlines without physical protection to prevent infection, or savings and healthcare in case of infection. Thinking down the line, those of low socioeconomic status are in danger of being unable to afford groceries, childcare, or even housing-possibly leading to eviction or dependence on government shelters. Unmistakably, the COVID-19 pandemic has had the effect of stratifying society, so it remains highly important to continue to consider the most vulnerable populations during this global health disaster. 


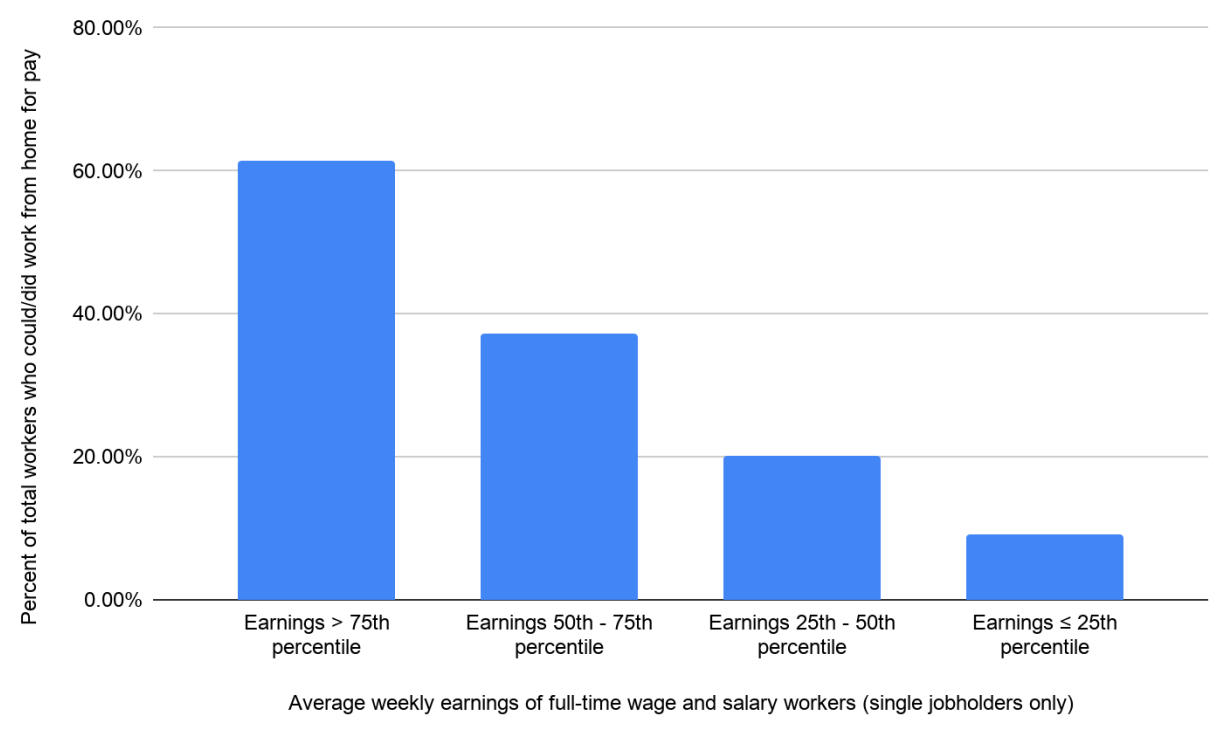

Figure 2. Ability to Work from Home Based on 2017-2018 Income Level. Adapted with permission from Functional Food Center, 2020, pp. 232 [5]

In terms of racial and ethnic identities, minorities, such as African-Americans and Hispanics, are among the most affected by SARS-CoV-2 proportional to population size in the United States [10]. The bar graph below breaks down fatalities by race or ethnicity to show that while Asian and White populations live in New York City in higher proportions, Black and Hispanic populations are dying of COVID-19 in higher proportions. There are many reasons for this disparity: racial minorities (especially those who are undocumented or have been institutionalized) are more likely in the United States to not have health insurance, disproportionately affected by poverty [11], more likely to live in urban areas [12], experience racism in the healthcare system, and be much less likely to be able to work

Population Percentage

COVID-19 Fatalities Percentage

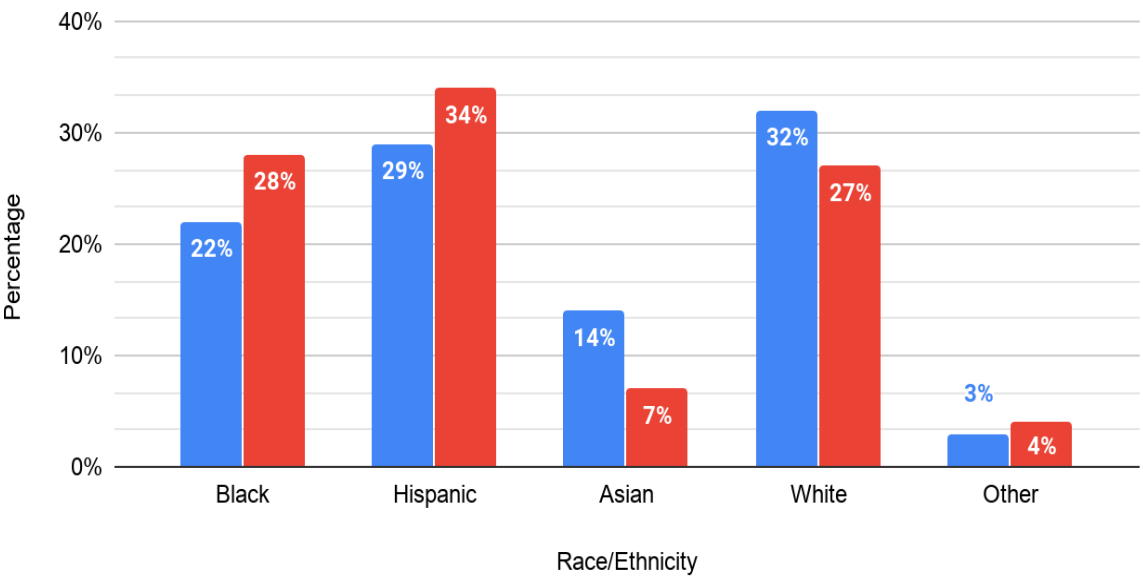

Figure 3. COVID-19 Fatalities by Race/Ethnicity in New York City. Adapted with permission from Functional Food Center, 2020, pp. 233 [5] 
from home [9]. The intersectionality between low socioeconomic status and minority status mean that the individuals that suffer from the pandemic the most are those who hold both or multiple risk-factor identities (e.g., minority elderly or minority urban homeless). It is important to identify intersectionalities as risk-factors for wellness or illness without using them as an excuse to exhibit stereotyping or victimization as a policymaker, healthcare provider, workplace executive, or public health organization.

Social disparities have a multitude of consequences for SARS-CoV-2 infection health effects, even without considering the effects of nutrition and immune function as this article aims to. This article defines the most vulnerable as populations that are elderly, low-income, urban, or part of a racial minority. Each of these intersectionalities will be considered when observing health patterns and offering nutritional guidance during the COVID-19 pandemic.

Nutrition Accessibility: Apart from being at-risk for COVID-19 due to societal factors, many of these groups are also the most jeopardized due to nutrition deficiencies - which can lead to other health-issuescompounding both incidence and symptomatology of COVID-19. Groups such as the elderly or ill are less likely to have access to fresh foods, due to not having availability or ability to obtain transportation to grocery stores. Many older people in the United States also live in nursing homes, which, due to a strict business model and high volumes, prepare residents' meals in a commercial kitchen with subpar products to fit a specific budget [13]. The same issues plague other institutionalized residents, such as those in detainment camps, jails and prisons, and mental health facilities, who have very poor nutritional status, in addition to already being in danger of contracting the deadly virus [14]. The nutritional access of these groups is not prioritized in the society and finding anything to eat is often difficult enough. Urban occupants, especially, may live in food deserts where their geographics-distance from farms or gardens with fresh harvests-limit them from accessing healthy and nutritional foods [15]. Moreover, as discussed in the previous section, minority or low-income groups are often continued to be called into non-remote or frontline work instead of being able to safely quarantine at home [9]. Due to busier schedules, workers deemed "essential" may be less able to find time to go grocery shopping or cook for their families to provide nutritious meals. Furthermore, lack of education or healthcare counseling for members of these populations may mean that they do not understand the importance of day-to-day nutrition and physical activity. All these listed effects already existed before the pandemic.

Quarantines and stay-at-home orders imposed during the COVID-19 pandemic further complicate the accessibility issue: people are prevented from using public transportation, which they may have relied on, cannot find effective personal protective equipment (PPE) to wear outside of the home, don't 
get regular physical activity, or are living with greater numbers of people in one household. Many are forced to survive on foods that are cheaper or can be stored for longer periods of time, which are more likely to contain preservatives and high amounts of salts and sugars - which can lead to complications such as obesity, hypertension, and diabetes [16]. While some of these factors increase likelihood of exposure to viral disease and others increase symptom severity after the disease is already contracted, all of them contribute to a lack of general nutritional wellness especially in vulnerable populations that can lead to poor health outcomes, which only multiply during a pandemic.

Diet Effects on Comorbidities: Disparities in SARSCoV-2 risk and general, long-term nutrition education and accessibility in elderly, low-income, urban, and racial minority groups can play a role in leading to reliance on processed and fast foods, high in saturated fats, sugars, and refined carbohydrates. Malnutritious diets place workers and families at an increased risk for dangerous, often lethal chronic diseases such as obesity, hypertension, diabetes, and cardiovascular disease [16]. Conditions like these are often irreversible and expensive to treat or control. However, these chronic diseases may also be responsible for another deadly consequence. Chronic disease serves as a risk factor for viral infections.

Although COVID-19 and other viral diseases are primarily fought using pathogen destruction models, the incidence of comorbidities in this pandemic highlights the importance of considering the holistic metabolic processes of the body. The most common metabolic diseases such as type 2 diabetes and hypertension are associated with the most severe COVID-19 symptoms due to multiorgan damage leading to heightened inflammation, toxic metabolite buildup, muscle weakness, thrombotic risk, respiratory decline, and possibly eventual death [17]. Those that survive have a tougher time re-reaching their preinfection health.

Therefore, the most susceptible to SARS-CoV-2 are those affected by prior chronic conditions, which are unfortunately also likely to appear in older age, adding another layer of risk for the elderly. In fact, data from New York state determined that $89.8 \%$ of fatalities from the disease had at least one comorbidity [6]. Comorbidities are common in cohorts of SARS-CoV-2 patients as shown in Graph 3 below, with the most common being hypertension, diabetes, and heart disease (including hyperlipidemia, coronary artery disease, atrial fibrillation, and stroke which are all primarily pathogenic in the cardiovascular system). These chronic diseases place individuals at an increased risk for contracting SARS-CoV-2 and severely worse health outcomes even if recovered due to low systemic immunity and multi-system damage $[16,18]$. Nutrition initiatives considering foods' abilities in exacerbating or managing chronic disease should be placed on high priority for healthcare providers and policymakers when working with vulnerable societies. 


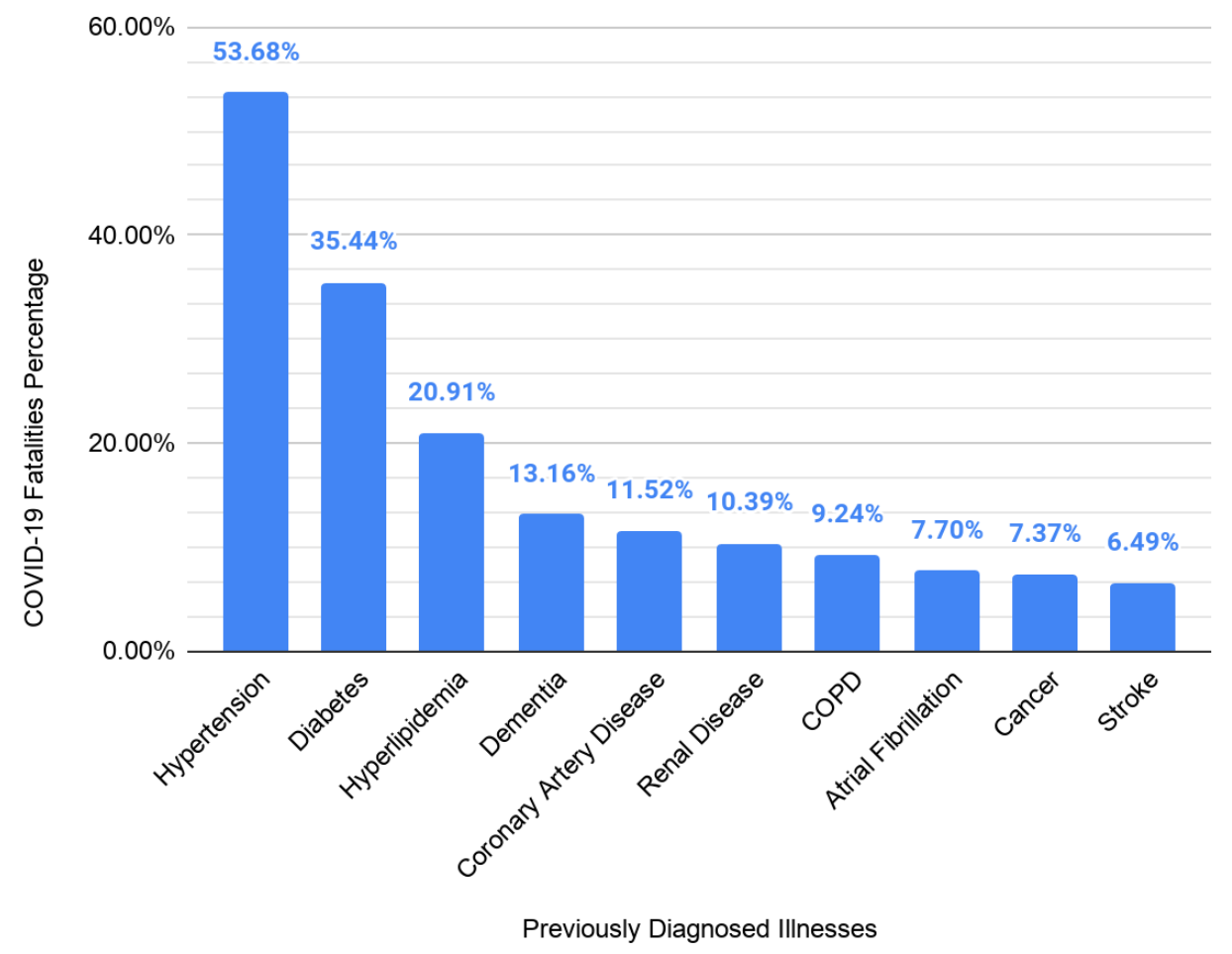

Figure 4. New York State Comorbidities in COVID-19 Fatalities. Adapted with permission from Functional Food Center, 2020, pp. 234 [5]

Diet Effects on Immunity: Aside from having a collateral effect, issues in diet can also harm the immune system directly. Although nutrition has a huge effect on comorbidities as discussed above, it also can more directly affect susceptibility to the SARS-CoV-2 virus. Good nutrition is invaluable in providing the body with the means to prevent viral transmission and promote disease recovery [19]. Both too much and too little of various dietary compounds can harm the immune system and prevent it from fighting off foreign dangerous viruses. At this time, although many longterm clinical studies have not been done regarding SARS-CoV-2, numerous studies in the past have been found to demonstrate the relationship of food consumption to immunity and coronaviruses. For example, an excess in fat intake, leading to elevations in free fatty acids and triglycerides in the body, can depress the immune system $[17,20]$. On the other hand, deficiencies in functional foods such as macronutrients, like proteins or some fatty acids, or micronutrients, like vitamins and minerals, are also found to considerably harm the immune system [20$22]$. These issues in nutrition can create barriers to immune cell triggering and function or inflammation control, leading to worsening of symptoms after initial exposure to the virus.

Many beneficial dietary compounds are most easily found in non-packaged foods, such as fresh products like fruits and vegetables that can be found in grocery stores. To demonstrate, one study found that a group of old-aged participants had a much better antibody response to vaccination against respiratory infections-like the ones caused by the COVID-19 disease-such as pneumonia and meningitis if they consumed 5 portions of fruits and vegetables a day compared to a group that only consumed 2 portions 
daily [23]. However, this type of diet is not attainable for many facing nutrition accessibility issues, resulting in a huge disadvantage to the groups of people that may contract SARS-CoV-2 virus or the groups of people that are least able to get better. The solution may arise in improving education and accessibility or finding substitutes to these nutritious dietary compounds for endangered populations.

Recommendations: Despite identifying foods that can be used as prevention and to act against the SARS-CoV2 virus, the biggest problem at hand is in getting advice and bioactive foods to vulnerable populations. This is a task for public health officials and food scientists, who can play a big role in advocating for consumption and accessibility of these foods [5]. We proceed here to a compilation of possible guidance and suggestions for adequate accessibility and consumption of specific compounds to build a healthy diet during a pandemic:

- Improve nutrition options in high-volume areas such as schools, nursing homes, homeless shelters, and soup kitchens. Lowincome children who relied on school lunches as their most nutritious meal of the day are deprived during quarantine and school closures. Nursing homes are largely responsible for the health of their elderly and frail residents so nutritious meals become more important. Lastly, with the growing rates of eviction and bankruptcy during the pandemic, homeless shelters and soup kitchens are at higher capacity than ever, but many of their staff and volunteers are either unable or unwilling to work due to dangers posed by the virus. Increasing funding, staffing, and food budgets in these three mentioned locations, as well as others, can dramatically improve low-income citizens' diets and therefore, health.
- Adolescent and adult nutrition education programs: populations including the elderly, low-income, urban, or racial minorities should be empowered to make their own health decisions based on the knowledge they are given. This will change the way constituents think about food or exercise and create lasting health changes throughout lifetimes rather than temporary dependence on providers and dieticians during the pandemic. Long-term nutrition and chronic disease management can create priceless opportunities for healthy lifestyles in vulnerable populations.

- Establish better grocery access through either meal delivery or transportation initiatives. Since fresh foods, rather than processed foods, contain higher levels of immunity improving bioactive compounds, the burden of frequent grocery trips should be offset for vulnerable populations. For those quarantining or unable to drive to the grocery store, this plan could improve the frequency of consumption of fresh foods. Additionally, accessible locations that can host temporary produce and farmers markets should be prioritized in food desert areas. Urban citizens may be able to go grocery shopping more often and purchase fresh foods that are more nutrient-dense.

- Reduce prices on healthy foods in supermarkets to make nutrient-rich foods more easily attainable for low-income or racial minorities. This recommendation may be more difficult to implement as it is largely dependent on agriculture and transportation sectors to reduce prices. Governmental action may also help by allowing for reduction of taxes on foods or by expanding eligibility for unemployment or food stamp benefits. 
- Develop affordable processed functional foods fortified with elusive but essential bioactive compounds that are effective in the immune system and multi-organ health: including proteins, antioxidants, probiotics, lipids, and more [24]. Functional foods are "natural or processed foods that contain biologically-active compounds; which, in defined, effective, and non-toxic amounts, provide a clinically proven and documented health benefit utilizing specific biomarkers for the prevention, management, or treatment of chronic disease or its symptoms" [25]. For cases in which it is impractical to obtain daily recommended amounts of essential nutrition through fresh fruits, vegetables, and other foods, processed functional foods can be important discoveries in an accessible and nutritious diet. For the most vulnerable populations-to prevent both malnutrition and COVID-19-food scientists should work to develop and market fortified supplements and food items.

Especially in the wake of an imminent financial crisis, economics and public health are closely intertwined [26-27]. All these suggested initiatives require funding and support, as well as fast action and innovation as the pandemic progresses, from the government, public health organizations, and scientific researchers.

\section{CONCLUSION}

Ultimately, populations most vulnerable to COVID-19 due to societal structures are also vulnerable to nutrition deficiencies and the associated chronic diseases and immunity decline. For vulnerable urban, low-income, old age, institutionalized, or racial minority individuals, it is of utmost importance to continue to consider nutrition as a driving factor in protecting against infectious disease contraction or mortality. Further clinical studies must be done to establish support for use of these and other compounds against SARS-CoV-2 infection, but recommendations for bioactive compounds and functional foods are an excellent method to create a prophylactic nutritional plan to improve patients' and populations' immune function and prevention of multisystem (especially respiratory) damage during this airborne viral pandemic. Structural recommendations (such as those in policy, public health, education, urban planning, economics, etc.) may also be used to improve accessibility to essential nutrition and healthy diets. This article aims to study the relationship between the factors of SARS-CoV-2 viral infection, health disparities by population, and nutrition to supply non-pharmaceutical recommendations to fight the SARS-CoV-2 virus. The findings provided are invaluable in working to alleviate the health disparities and negative outcomes due to the COVID-19 pandemic.

Abbreviations: Coronavirus disease 2019 (COVID-19), Severe acute respiratory syndrome coronavirus 2 (SARS-CoV-2), personal protective equipment (PPE), Supplemental Nutrition Assistance Program (SNAP)

Competing Interests: The author have no conflicts of interest to disclose.

Funding: The author declare no external funding.

\section{REFERENCES:}

1. Eurosurveillance Editorial T: Note from the editors: World Health Organization declares novel coronavirus (2019$\mathrm{nCoV}$ ) sixth public health emergency of international concern. Euro Surveill 2020, 25. https://doi.org/10.2807/15607917.ES.2020.25.5.200131e

2. "Coronavirus Cases:" Worldometer, Accessed September 10, 2020. www.worldometers.info/coronavirus/\#countries

3. Grasselli G, Zangrillo A, Zanella A, et al: Baseline Characteristics and Outcomes of 1591 Patients Infected 
With SARS-CoV-2 Admitted to ICUs of the Lombardy Region, Italy. JAMA 2020, 323(16): 1574-1581. https://doi.org/10.1001/jama.2020.5394

4. Pinti M, Appay V, Campisi J, Frasca D, Fülöp T, Sauce D, Larbi A, Weinberger B, Cossarizza A: Aging of the immune system: focus on inflammation and vaccination. European journal of immunology 2016, 46(10): 2286-301. https://doi.org/10.1002\%2Feji.201546178

5. Functional Food Center, Martirosyan D: Functional Foods and Viral Diseases. Food Science Publisher 2020, Volume 8: 229-252.

6. New York State Department of Health: "Fatalities" NYS COVID19 Tracker, Accessed June 1, 2020.

https://covid19tracker.health.ny.gov/views/NYSCOVID19-Tracker/NYSDOHCOVID-19Tracker-Fatalities?\% 3Aembed=yes\&\%3Atoolbar=no\&\%3Atabs $=n$

7. Borowiak M, Ning F, Pei J, Zhao S, Tung HR, Durrett R: Controlling the spread of COVID-19 on college campuses. arXiv preprint arXiv:2008.07293. 2020.

8. Desjardins MR, Hohl A, Delmelle EM: Rapid surveillance of COVID-19 in the United States using a prospective space-time scan statistic: Detecting and evaluating emerging clusters. Applied Geography 2020, 102202. https://doi.org/10.1016\%2Fj.apgeog.2020.102202

9. U.S. Department of Labor: JOB FLEXIBILITIES AND WORK SCHEDULES - 2017-2018 DATA FROM THE AMERICAN TIME USE SURVEY. Bureau of Labor Statistics 2019. https://www.bls.gov/news.release/pdf/flex2.pdf

10. Laurencin CT, McClinton A: The COVID-19 Pandemic: A Call to Action to Identify and Address Racial and Ethnic Disparities. J. Racial and Ethnic Health Disparities 7 2020: 398-402. https://doi.org/10.1007/s40615-020-00756-0

11. Firebaugh G, Acciai F: For blacks in America, the gap in neighborhood poverty has declined faster than segregation. Proceedings of the National Academy of Sciences 2016, 113(47): 13372-13377. https://doi.org/10.1073/pnas.1607220113

12. Copeland VC: African Americans: Disparities in Health Care Access and Utilization, Health \& Social Work 2005, 30(3): 265-270, https://doi.org/10.1093/hsw/30.3.265

13. Strathmann S, Lesser S, Bai-Habelski J, Overzier S, PakerEichelkraut HS, Stehle $\mathrm{P}$, Heseker $\mathrm{H}$ : Institutional factors associated with the nutritional status of residents from 10 German nursing homes (ErnSTES study). The journal of nutrition, health \& aging 2013, 17(3): 271-6. https://doi.org/10.1007/s12603-012-0410-8

14. Carney MA: Border meals: Detention center feeding practices, migrant subjectivity, and questions on trauma. Gastronomica: The Journal of Food and Culture 2013, 13(4): 32-46

15. Dubowitz T, Zenk S, Ghosh-Dastidar B, Cohen D, Beckman R, Hunter G, Collins R. Healthy food access for urban food desert residents: Examination of the food environment, food purchasing practices, diet and BMI. Public Health Nutrition 2015, 18(12); 2220-2230. https://doi.org/10.1017/S1368980014002742

16. Butler MJ, Barrientos RM: The impact of nutrition on COVID-19 susceptibility and long-term consequences. Brain, behavior, and immunity 2020. https://doi.org/10.1016/j.bbi.2020.04.040

17. Ayres JS. A metabolic handbook for the COVID-19 pandemic. Nat Metab 2, 572-585; 2020. https://doi.org/10.1038/s42255-020-0237-2

18. Liang W, Guan W, Chen R, Wang W, Li J, Xu K, ... Li S: Cancer patients in SARS-CoV-2 infection: a nationwide analysis in China. The Lancet Oncology 2020, 21(3): 335337. https://doi.org/10.1016/\$1470-2045(20)30096-6

19. Romano L, Bilotta F, Dauri M, Macheda S, Pujia A, De Santis GL, ... De Lorenzo A: Short Report-Medical nutrition therapy for critically ill patients with COVID-19. European Review for Medical and Pharmacological Sciences 2020, 24: 4035-4039.

https://doi.org/10.26355/eurrev 202004 20874

20. Gleeson M, Nieman DC, Pedersen BK: Exercise, nutrition and immune function, Journal of Sports Sciences 2004, 22(1): $115-125$ https://doi.org/10.1080/0264041031000140590

21. Naja F, Hamadeh R: Nutrition amid the COVID-19 pandemic: a multi-level framework for action. Eur J Clin Nutr 2020. https://doi.org/10.1038/s41430-020-0634-3

22. Nonnecke BJ, et al: "Acute phase response elicited by experimental bovine diarrhea virus (BVDV) infection is associated with decreased vitamin $D$ and $E$ status of vitamin-replete preruminant calves." Journal of dairy science 2014, 97(9): 5566-5579. https://doi.org/10.3168/jds.2014-8293

23. Gibson A, Edgar JD, Neville CE, Gilchrist SE, McKinley MC, Patterson CC, Young IS, Woodside JV: Effect of fruit and vegetable consumption on immune function in older people: a randomized controlled trial. The American journal of clinical nutrition, 2012. 96(6):1429-36.

24. Polamarasetti $P$, Martirosyan D: Nutrition planning during the COVID-19 pandemic for aging immunity. Bioactive Compounds in Health and Disease, 2020. 3(7):109-23. https://doi.org/10.31989/bchd.v3i7.733

25. Gur J, Mawuntu M, Martirosyan D: FFC's Advancement of Functional Food Definition. Functional Foods in Health and Disease. 2018, 8(7): 385-97.

26. Galanakis CM: The Food Systems in the Era of the Coronavirus (COVID-19) Pandemic Crisis. Foods. 2020, 9(4): 523. https://doi.org/10.3390/foods9040523

27. Dharmasena S, Bessler DA, Capps Jr O: Food environment in the United States as a complex economic system. Food Policy 2016, 61: 163-75.

https://doi.org/10.1016/j.foodpol.2016.03.003 